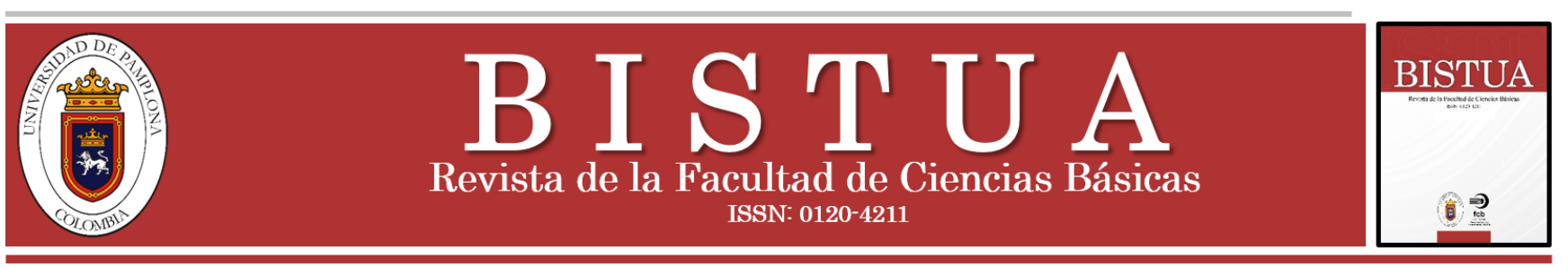

\title{
Estimación del potencial de calentamiento global en un sistema de potabilización de un acueducto municipal en Colombia
}

\section{Estimation of the Global Warming Potential in a Water Treatment System of a Municipal Aqueduct in Colombia}

\author{
Martha Lucía Barrera Pérez ${ }^{\text {a }}$ Omar Alberto Ávila ${ }^{\mathrm{b}}$. Ligia Beleño Montagut ${ }^{\mathrm{c}}$ \\ ${ }^{a}$ Departamento de matemáticas y ciencias naturales, Universidad Autónoma de Bucaramanga, Colombia. \\ ${ }^{b}$ Escuela de Ingeniería Ambiental y de Saneamiento, Instituto Universitario de la Paz - UNIPAZ, Colombia. \\ ${ }^{c}$ Departamento de matemáticas y ciencias naturales, Universidad Autónoma de Bucaramanga, Colombia. \\ Contacto:mbarrerp@unab.edu.co
}

Recibido: Marzo 10, 2020. Aceptado: Junio 30, 2020

https://doi.org/10.24054/01204211.v1.n1.2020.4170

\section{Resumen}

Este este estudio presenta los resultados de la evaluación de impactos ambientales (IA) asociados a la potabilización de $1 \mathrm{~m}^{3}$ de agua cruda, en dos plantas de tratamiento de agua potable (PTAP) del Acueducto Metropolitano de Bucaramanga (AMB), con y sin bombeo, en la etapa de distribución. La metodología aplicada fue el Análisis de Ciclo de Vida según la NTC-ISO14040/14044:2007, uso del software SimaPro 9.0, método de evaluación IMPACT 2002+ V2.15 y el IPCC (lntergovernamental Panel on Climate Change) 2013 GWP 20a V1.03. De la evaluación ambiental, se obtuvo que el mayor IA fue la ecotoxicidad acuatica en $75,8 \%$ por presencia de aluminio y acrilamida en $95 \%$ y $4 \%$; seguido del calentamiento global, energía no renovable y no cancerigenos en un $6 \%$ cada impacto, en relación a la emisión de $\mathrm{CO}_{2}, \mathrm{NO}_{2}, \mathrm{SO}_{2}$ y material particulado en $62 \%, 18 \%, 8,3 \%$ y $12 \%$ respectivamente. Finalmente, se determinó el potencial del calentamiento global para la PTAP sin bombeo en $0,10 \mathrm{~kg}$ $\mathrm{CO}_{2 \mathrm{eq}} / \mathrm{m}^{3}$ agua potable y de $0,51 \mathrm{~kg} \mathrm{CO} 2 \mathrm{eq} / \mathrm{m}^{3}$ agua potable para la PTAP con bombeo. La evaluación realizada analizó los efectos de las emisiones a corto plazo en un periodo de integración de 20 años, así como la predicción de los efectos de los GEI (gases de efecto de invernadero) a largo plazo en tiempos largos de integración a 100 y 500 años.

Palabras clave: ACV; Calentamiento Global; GEI; PTAP.

\section{Abstract}

This this study presents the results of the evaluation of environmental impacts (EI) associated with the purification of 1 $\mathrm{m}^{3}$ of raw water, in two drinking water treatment plants (PTAP) of the Metropolitan Aqueduct of Bucaramanga (AMB), with and without pumping, in the distribution stage. The methodology applied was the Life Cycle Analysis according to NTC-ISO14040/14044: 2007, use of SimaPro 9.0 software, IMPACT 2002+ V2.15 and IPCC (Intergovernmental Panel on Climate Change) 2013 GWP 20a V1.03 evaluation method. From the environmental evaluation, it was obtained that the highest EI was aquatic ecotoxicity in $75.8 \%$ due to the presence of aluminum and acrylamide in $95 \%$ and $4 \%$; followed by global warming, nonrenewable and non-carcinogenic energy in $6 \%$ each impact, in relation to the emission of $\mathrm{CO}_{2}, \mathrm{NO}_{2}, \mathrm{SO}_{2}$ and particulate matter in $62 \%, 18 \%, 8.3 \%$ and $12 \%$ respectively. Finally, the global warming potential for the PTAP without pumping was determined at $0.10 \mathrm{~kg} \mathrm{CO} 2 \mathrm{eq} / \mathrm{m}^{3}$ drinking water and $0.51 \mathrm{~kg} \mathrm{CO} \mathrm{CO}_{2 \mathrm{q}} / \mathrm{m}^{3}$ drinking water for the PTAP with pumping. The evaluation of the effects of short-term emissions in an integration period of 20 years was carried out, as well as the prediction was obtained. of the long-term effects of GHGs (greenhouse gases) in long integration times at 100 and 500 years

Keywords: ACV; Global Warming; greenhouse gases; drinking water treatment plant.

\section{Introducción}

Desarrollar una red de agua potable y alcantarillado, requiere del consumo de energía eléctrica en las etapas de captación del agua de fuentes subterráneas o superficiales, como del tratamiento (aireación, inyección de aditivos químicos, etc.), distribución y transporte desde la misma hacia el consumidor (algunos procesos más complejos requieren mayor consumo energético) [3].

Por lo anterior, se resalta la importancia de la mayor carga ambiental atribuida al consumo de energía eléctrica, específicamente en el proceso de potabilización siempre y cuando el sistema de bombeo por captación o distribución superen los consumos de producción. Por lo tanto, abordar la mitigación en la región respecto a los impactos ambientales asociados por consumos energéticos y uso de sustancias químicas, implica conocer el estado de información que tienen los diversos actores de la población, respecto a la generación de emisiones de GEI directos e indirectos que conllevan al calentamiento global y, por consiguiente, al cambio climático, los cuales requieren del planteamiento de acciones por las entidades gubernamentales, según los 
resultados de este estudio [12]. Con relación a los planes de mitigación, "es necesario la elaboración de estudios especializados que identifiquen las actividades que aportan los GEI y se sugiere la incorporación de un sistema de identificación, seguimiento y control a la emisión de los mismos, con la articulación de la CDMB, CORPONOR y LA CAS y el Nodo Norandino de cambio climático, como de espacios institucionales que tienen incidencia en la gestión ambiental del territorio" [5].

Alrededor del tema se han realizado trabajos para analizar los impactos ambientales generados por un sistema de provisión de agua potable. Con relación a estudios previos se puede establecer que desde el año 2000, comienzan a reportarse trabajos en el marco de Análisis de Ciclo de Vida, para evaluar impactos ambientales de los sistemas de agua urbanos. Un primer trabajo publicado por [13], en el cual realiza la comparación de diferentes métodos de análisis de impacto por ciclo de vida para ecotoxicidad acuática, seguido por [10] quien desarrolló un procedimiento de evaluación del ciclo de vida para sistemas de agua urbanos en Suecia. Este estudio desarrolló un procedimiento iterativo que combina resultados empíricos y un marco analítico basado en la metodología ACV, usado en dos casos de estudio particulares: dos sistemas de agua urbana en dos regiones diferentes, una desarrollada (Göterborg, Suecia) y otra en desarrollo (King William's Town, Sur África); que permitieron analizar los indicadores de sostenibilidad ambiental para cada estudio.

Otro trabajo en el que utilizó el Análisis de Ciclo de Vida como una herramienta de gestión ambiental, considerando dos sistemas de producción de agua potable diferentes, utilizó, un sistema convencional que involucra procesos de pre-ozonización, adición de químicos, floculación, sedimentación, filtración, ozonización, cloración y almacenamiento como primer sistema; y el segundo, basado en el uso de una tecnología Sur Africana de filtración por membrana, seguido por procesos de pre-filtración, cloración y almacenamiento[4]. De acuerdo a la metodología $\mathrm{ACV}$, se enumeran las entradas y salidas (materias primas y energía) y se cuantifican los productos, subproductos y emisiones al aire, agua y suelo) para los dos sistemas. Este inventario genera diferentes impactos al medio ambiente según el tipo de categoría (calentamiento global, agotamiento de la capa de ozono por emisión de gases de efecto invernadero, formación de smog, acidificación, eutrofización por enriquecimiento de nutrientes, eco-toxicidad y toxicidad humana).

Otro referente importante aplica exitosamente la metodología de Análisis de Ciclo de Vida para la planeación estratégica del sistema de producción de agua potable en Sídney (Australia), con el fin de tomar decisiones metodológicas sobre el nivel de detalle requerido en cada una de las etapas del proceso [9].
Trabajos más recientes como el de [2] en Canadá, quien desarrolla un Análisis de Ciclo de Vida comparativo de plantas de tratamiento de agua potable: una planta de tratamiento convencional y una planta de nano filtración. Uno de los trabajos más recientes fue publicado por [14] en Noruega, en el que se presenta el Análisis de ciclo de vida de un sistema de agua potable y de aguas residuales en Trondheim, Noruega.

Para finalizar este acercamiento a la literatura, relacionada con la aplicación de la metodología ACV, aplicada a los sistemas de tratamiento de agua potable, es importante referenciar un trabajo reciente, desarrollado en Francia [8], dado que en la mayoría de estudio de ACV para los sistemas de producción de agua potable, no muestran con claridad la contribución al impacto ambiental, de los procesos unitarios relacionados con la infraestructura de la planta y con el tratamiento de lodos. Los resultados del análisis muestran en el diagrama de puntuación única, que el mayor impacto ambiental es producido por el consumo de combustibles fósiles, directamente relacionados con el consumo de electricidad y la producción de carbón activado.

El consumo de agua potable en la ciudad de Bucaramanga y en general en el país, ha tomado importancia, a partir de la problemática que han mostrado reportes relacionados en distintos sectores, con relación al impacto ambiental que genera la producción de agua potable, desde su captación hasta la distribución para su consumo. Lo anterior hace relevante un análisis de cuantificación del impacto ambiental sobre el proceso de potabilización, mediante la medición de distintas variables, en éste caso se analizó el consumo de energía eléctrica.

El trabajo presentado forma parte de los resultados de un proyecto de investigación realizado en la Universidad Industrial de Santander, de acuerdo a la información suministrada por el Acueducto Metropolitano de Bucaramanga. En el análisis del estudio, se evalúo el impacto ambiental potencial y el poder de calentamiento, de dos plantas de tratamiento de agua potable administradas por la entidad en mención y que abastecen del producto a las ciudades de Bucaramanga y Floridablanca.

Los sistemas estudiados cumplen con la función de dar tratamiento a un afluente de agua cruda superficial, proveniente de la Cuenca del Río Suratá y de la Microcuenca del Río Frio, a partir de un proceso mecánico, biológico y químico. El sistema de potabilización se compone por dos plantas que funcionan de forma independiente, las cuales cuentan con sistema de bombas para dosificación de sulfato. Del estudio se excluyó las cargas ambientales de la red de distribución de consumo, transporte de materias primas y los insumos requeridos en laboratorios y maquinarias. A continuación, se describen las dos Plantas de Tratamiento de Agua Potable (PTAP). 
La PTAP Bosconia está localizada en la vía que conduce de Bucaramanga al municipio de Matanza, diseñada con una capacidad de $2000 \mathrm{l} / \mathrm{s}$ de tipo convencional, conformado por procesos de pre-dosificación de químicos para coagulación, pre-sedimentación, canaleta Parshall, mezcla rápida, floculación mecánica, sedimentación, filtración, desinfección con cloro gaseoso y almacenamiento de $10000 \mathrm{~m}^{3}$ de capacidad. Esta planta cuenta con un sistema propio de Bombeo para distribución. Ver Figura 1.

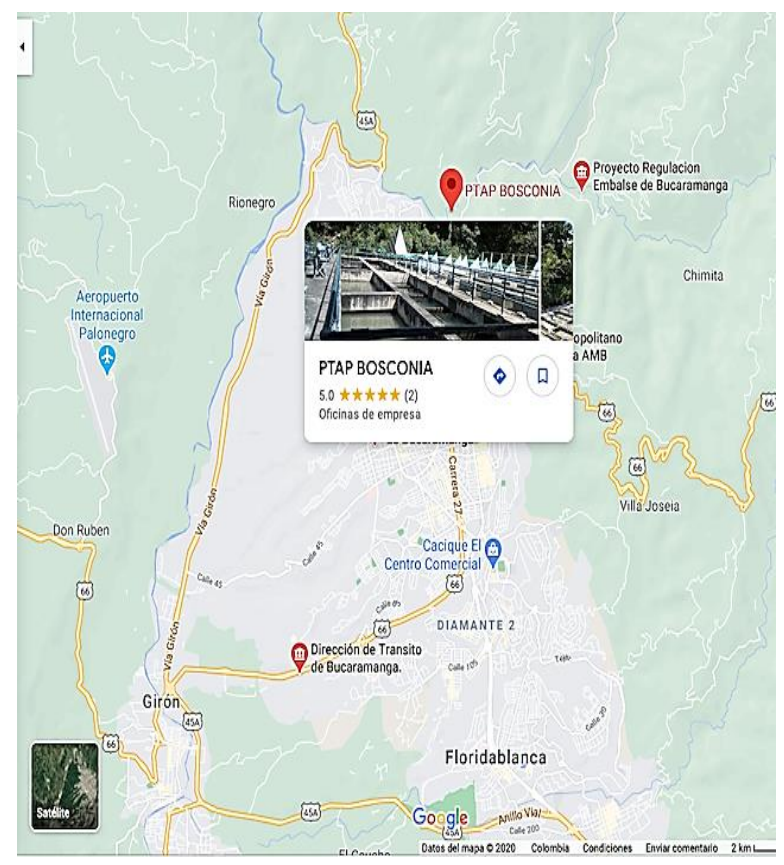

Figura 1. Ubicación PTAT Bosconia Fuente: Google Maps

La PTAP Floridablanca está localizada en la zona suroriental del Área Metropolitana de Bucaramanga, en la parte alta de los barrios Bucarica y Caracolí del municipio de Floridablanca, a una altura media de $1042 \mathrm{~m}$ sobre el nivel del mar. Dentro de los componentes estructurales, se encuentra: una canaleta Parshall, sistema de mezcla rápida, floculación hidráulica y mecánica, área de sedimentación y filtración, dos tanques de almacenamiento de coagulante y dos tanques de almacenamiento de agua tratada con capacidad de $1600 \mathrm{~m}^{3}$ y un tercero de $2400 \mathrm{~m}^{3}$ [1]. Ver Figura 2.

\section{Método}

El uso de la herramienta metodológica $\mathrm{ACV}$, puede usarse para generar información sobre los impactos ambientales potenciales de un proceso, bien o servicio, como es el caso del sistema de tratamiento y provisión de agua potable, enmarcada en la estructura de la familia de normas ISO 14040/14044:2007 [11,15]. La definición de una unidad funcional estándar, facilita la comparación de diferentes sistemas, para la cual se empleó $1 \mathrm{~m}^{3}$ de agua tratada en las
PTAP objeto de estudio. Para la interpretación y análisis de resultados, se empleó el software SimaPro 9.0, método de evaluación IMPACT 2002+ V2.15 y el IPCC (lntergovernamental Panel on Climate Change) 2013 GWP $20^{\mathrm{a}}, 100^{\mathrm{a}}$ y $500^{\mathrm{a}}, \mathrm{V} 1.03[7]$

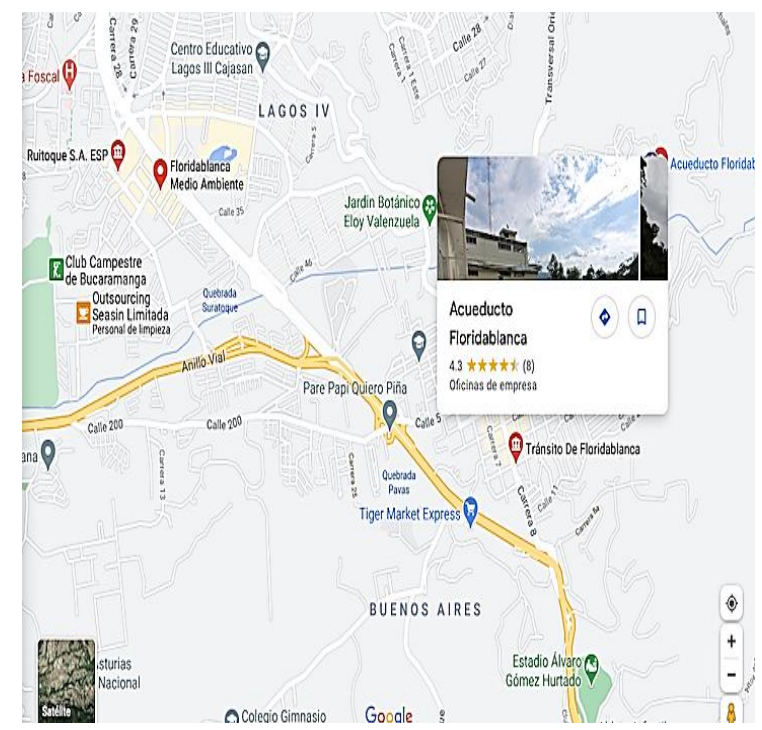

Figura 2. Ubicación PTAT Floridablanca Fuente: Google Maps

\section{Resultados}

Es importante resaltar que la emisión de $\mathrm{CO}_{2}$ equivalente suele calcularse habitualmente multiplicando la emisión de un GEI por su potencial de calentamiento global (PCG) en el plazo de 100 años y que en el caso de las mezclas de GEI se suman las emisiones de $\mathrm{CO}_{2}$ equivalente correspondientes a cada gas, siendo la emisión de dióxido de carbono equivalente una escala común para comparar las emisiones de diferentes GEI, aunque generalmente no existe ninguna conexión entre las emisiones de $\mathrm{CO} 2$ equivalente y las concentraciones de $\mathrm{CO} 2$ equivalente resultantes [6]. Por lo anterior, y en relación a los resultados obtenidos, se determinó una contribución del poder de calentamiento global a 100 años de $0.36 \mathrm{~kg} \mathrm{CO} 2 \mathrm{eq} / \mathrm{m}^{3}$ agua tratada en la PTAP Bosconia y de $0.09 \mathrm{~kg} \mathrm{CO} 2 \mathrm{eq} / \mathrm{m}^{3}$ agua tratada en la PTAP Florida

En la Tabla 1, se presenta los resultados obtenidos de la evaluación ambiental por consumo de requerimientos, el cual fue tomado como base, para la comparación de las dos unidades de proceso.

Como se puede observar en la tabla, la contribución del calentamiento global en promedio, estaá originado principalmente por el consumo de energía eléctrica en un 93\%, proveniente de la matriz eléctrica colombiana, seguida del aporte por uso combustible fósil $4 \%$ y la diferencia del impacto, atribuida al consumo de poliamida en $3 \%$. En la 
Tabla 2 se presenta la estimación del poder de calentamiento global en proyección a 20, 100 y 500 años.

Tabla 1. Resultados del poder del Calentamiento Global para el Sistema de Acueducto de Bucaramanga y su Área Metropolitano.

\begin{tabular}{lcc}
\hline $\begin{array}{c}\text { Requerimiento } \\
(\text { kg CO2eq) }\end{array}$ & $\begin{array}{c}\text { PTAP } \\
\text { BOSCONIA }\end{array}$ & $\begin{array}{c}\text { PTAP } \\
\text { FLORIDA }\end{array}$ \\
\hline Energía & 0,3519 & 0,0784 \\
\hline Combustible & 0,0108 & 0,0043 \\
\hline Poliamida & 0,0004 & 0,0058 \\
\hline Fuente: Autores & &
\end{tabular}

Tabla 2. Resultados de proyección del Poder del Calentamiento Global (20, 100 y 500 años) del Sistema de Acueducto de Bucaramanga y su Área Metropolitano.

\begin{tabular}{lcc}
\hline $\begin{array}{c}\text { Huella de Carbono } \\
\text { (kg CO2eq) }\end{array}$ & $\begin{array}{c}\text { PTAP } \\
\text { BOSCONIA }\end{array}$ & $\begin{array}{c}\text { PTAP } \\
\text { FLORIDA }\end{array}$ \\
\hline IPCC GWP 20 & 0,5150 & 0,1020 \\
IPCC GWP $100^{\mathrm{a}}$ & 0,3630 & 0,0884 \\
IPCC GWP 500 & 0,3090 & 0,0815 \\
\hline
\end{tabular}

Fuente: Autores

Los resultados presentados en la Tabla 2, evidencian un aporte de $0.52 \mathrm{~kg} \mathrm{CO} 2 \mathrm{eq} / \mathrm{m}^{3}$ agua tratada en la PTAP Bosconia, para un periodo de tiempo de 20 años. Así mismo, se presenta una disminución del $30 \%$ de la huella de carbono 80 años después y de $15 \%$ adicional, para un periodo de 500 años. En el caso de la PTAP Florida, se determinó un aporte de $0.09 \mathrm{~kg} \mathrm{CO} \mathrm{CO}_{2 \mathrm{eq}} / \mathrm{m}^{3}$ agua tratada a 20 años, con una disminución del $13 \%$ a 100 años y del $8 \%$ a 500 años.

\section{Discusión}

En la Figura 3 se presenta la medida de la totalidad de la emisión de gases de efecto invernadero en unidades de $\mathrm{kg}$ de

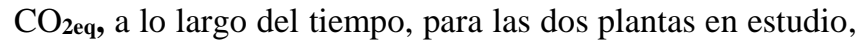
con una predicción a 20, 100 y 500 años. Para el cálculo de esta medida se multiplica la masa del gas por el potencial o poder de calentamiento global (GWP); dado que este factor disminuye con el tiempo, es de esperarse que la contribución al calentamiento global por efecto de gases de efecto invernadero, se reduzca con el paso de los años, por causa de la difusión de los mismos. Se puede observar en la Figura 3, que la mayor contribución ambiental para cada periodo evaluado es atribuida por la planta Bosconia. Lo anterior obedece al mayor consumo de energía eléctrica requerido en el proceso de captación del agua a la PTAP y la distribución para consumo. Respecto a la planta Floridablanca, esta presenta baja contribución al Calentamiento Global, dado que presenta menor consumo de energía eléctrica en sus etapas operativas, y la distribución del producto se hace por gravedad. La Figura 4, muestra la medida de la contribución al calentamiento global en $\mathrm{kg}$ de $\mathrm{CO}_{2 \text { eq }}$ a 20 años, para cada uno de los gases de efecto invernadero generados en cada una de las PTAP de estudio.

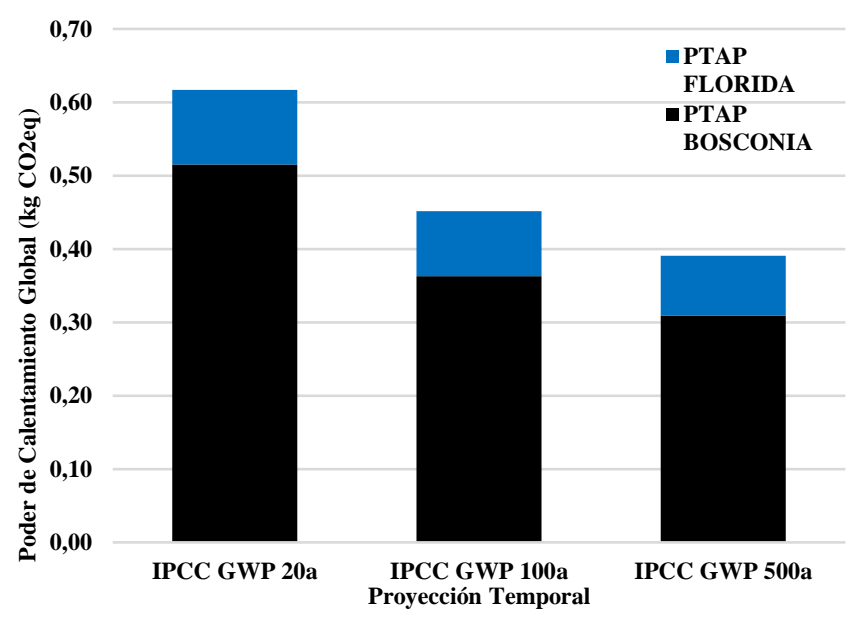

Figura 3. Contribución comparativa del poder del Calentamiento Global para las plantas de tratamiento de Agua Potable. Fuente: Autores

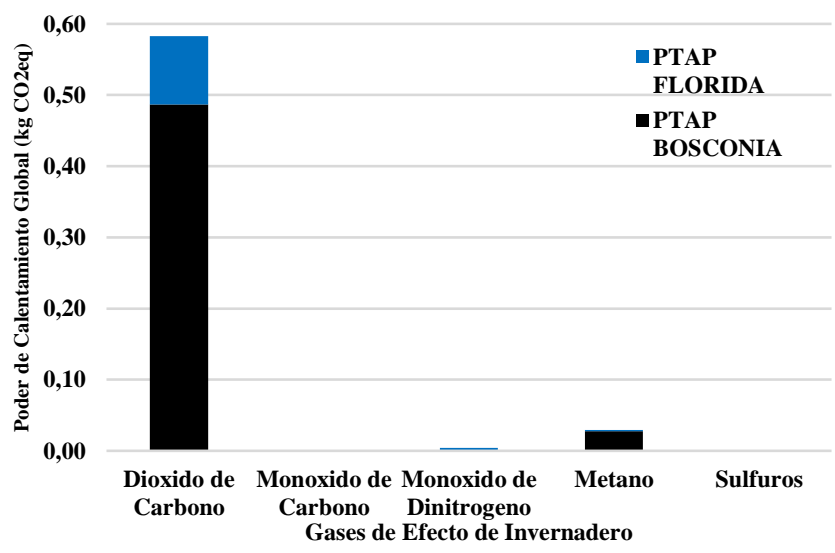

Figura 4. Poder del Calentamiento Global a 20 años Fuente: Autores

De la evaluación ambiental, se logró identificar el aporte promedio en $94 \%$ por la emisión de dióxido de carbono relacionado al consumo de energía eléctrica, seguido del metano en $4 \%$ asociado a la combustión de hidrocarburos en los motores de cogeneración, que utilizan las plantas para la distribución del producto. Así mismo, se evidencia el aporte ambiental al calentamiento global por la emisión del monóxido de di-nitrógeno, monóxido de carbono y sulfuros en $1.65 \%, 0.25 \%$ y $0.1 \%$, respectivamente.

Otro análisis realizado fue la contribución del calentamiento global por el uso de los diferentes recursos energéticos, tal como se muestra en la Figura 5. De la Figura 5 se evidencia para la PTAP Bosconia y Florida un aporte promedio del $96 \%$ y $88 \%$, asociado a la energía eléctrica, el cual tiene una disminución de hasta el $36 \%$ y $19 \%$ del impacto en un tiempo de 500 años. De igual forma, se analiza que por uso de combustible en la PTAP Bosconia y Florida, se estima un aporte del 3\% y 5\%, con una disminución del impacto $92 \%$ y $18 \%$ evaluados a 500 años. Por último, se 
determinó un aporte al calentamiento global del 1\% y $7 \%$ para la PTAP Bosconia y Florida, en relación al consumo de polímeros disminuyendo el impacto para un periodo de 500 años en $69 \%$ y $35 \%$, respectivamente.

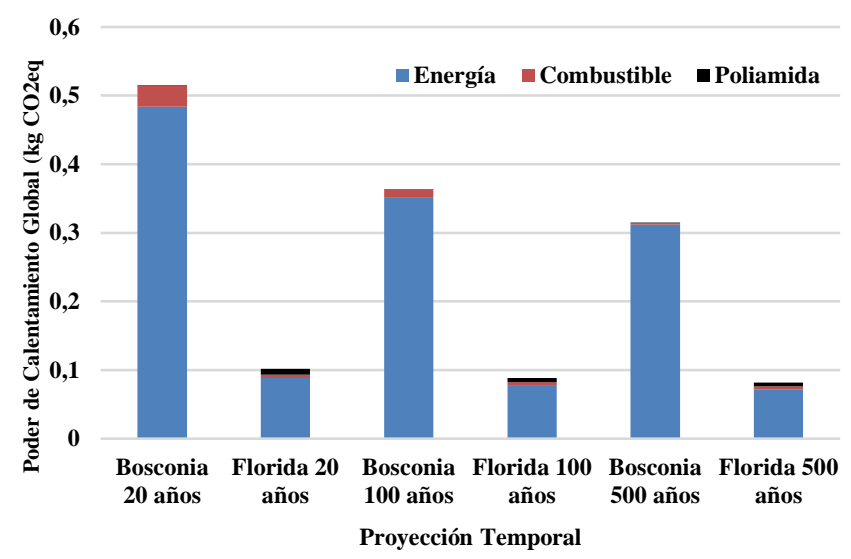

Figura 5. Poder del Calentamiento Global a 100 años Fuente: Autores

Finalmente, se obtuvo la evaluación ambiental global atribuida al proceso comparativo de las PTAP, tal como se muestra en la Figura 6.

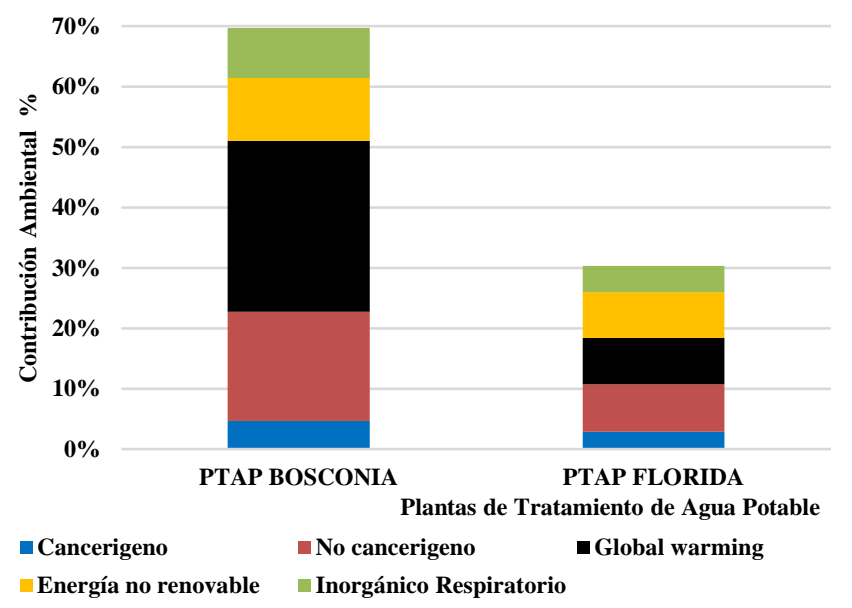

Figura 6. Potencial de Calentamiento Global a 500 años Fuente: Autores

De la evaluación ambiental representada en la Figura 6, se estima una contribución ambiental del $33 \%$ por calentamiento global, seguido del $26 \%$ por sustancias no cancerígenas, energía no renovable en $20 \%$, inorgánicos respiratorios en $13 \%$ y sustancias cancerígenas en $8 \%$ de la carga ambiental. El análisis comparativo muestra una reducción ambiental entre ambas plantas PTAP del 30\%, evidenciando el mayor impacto a la PTAP Bosconia $(74,53 \mathrm{uPt})$ y la PTAP Florida $(32,32 \mathrm{uPt})$.

\section{Conclusiones}

De la evaluación del poder de calentamiento global, se dedujo que el impacto ambiental se atribuye principalmente a las emisiones directas e indirectas de los GEI (94\% dióxido de carbono y $4 \%$ metano) generados por los consumos de recursos energéticos $(93 \%$ energía eléctrica y $4 \%$ combustibles fósiles).

De acuerdo con el análisis comparativo de las dos plantas de tratamiento de agua potable. puede observarse que, en un periodo de 20 años, la planta de Bosconia produce un impacto del $80 \%$ sobre el calentamiento global, debido al consumo de energía eléctrica en la etapa de la distribución, mayor a la PTAP Florida. Así mismo, se deduce que en un periodo de 500 años disminuye el calentamiento global en $40 \%$ para la PTAP Bosconia y 20\% para la PTAP Florida. Por lo anterior, se recomienda que para reducir el impacto la planta de tratamiento sea construida sobre la parte más alta de la ciudad de Bucaramanga, para que la distribución a la ciudad se haga por gravedad.

Del análisis ambiental global potencial, se enfatiza la importancia en la disminución de sustancias contaminantes tales como: dióxido de carbono, metano, óxidos de nitrógeno y azufre, material particulado, metales, entre otros, que conllevan a la activación de impactos potenciales como: calentamiento global, inorgánicos respiratorios, energías no renovables, sustancias cancerígenas y no cancerígenas.

\section{Reconocimientos}

Agradecimiento especial por sus contribuciones para esta investigaciòn al ingeniero Jhon Barreneche Jefe de PTAP Bosconia y a la ingeniera Yolanda Otero Jefe de PTAP Floridablanca.

\section{Referencias}

[1] Acueducto Metropolitano De Bucaramanga S.A. E.Sp. Capítulo 4: Aspectos Técnicos Y Operativos. [Book Section] // Evaluación Integral De Prestadoradores De Servicios Públicos Domiciliarios.. - Bogotá D.C. : [S.N.], 2014

[2] Bonton A. Et ál. et Comparative life cycle assessment of water treatment plants. [Journal]. - [s.l.] : ELSEVIER. Desalination, 2011. - Vol. 284., 42-54 .

[3] CEPAL - Serie Recursos Naturales e Infraestructura $\mathrm{N}^{\circ} 170$ Eficiencia energética y regulación económica en los servicios. Naciones Unidas, Santiago de Chile, enero de 2015 ISSN 16809017

https://repositorio.cepal.org/bitstream/handle/11362/37630/1/S1 $\underline{421127 \text { es.pdf }}$

[4] Friedrich E. Life-cycle assessment as an environmental management tool in the production of potable water. [Journal]. [s.1.] : Water Science and Technology., (2002). 46 (9), 29-36.

[5] Gomez A:; Salazar M. Cambio climático y mitigación: prospectiva para la región nororiental-santanderes. DELOS: Desarrollo Local Sostenible, 7 (19) (2014). 
https://eumed.net/rev/delos/19/cambio-climaticomitigacion.html

[6] IPCC.Calentamiento global de 1,5 $5^{\text {a }}$ (2019) Intergovernmental Panel on Climate Change. ISBN 978-92-9169-353-5 https://www.ipcc.ch/site/assets/uploads/sites/2/2019/09/SR15_S ummary Volume spanish.pdf

[7] IPCC. Kyoto, Mayo 18 (2019). Updates Methodology for Greenhouse Gas Inventories. https://www.ipcc.ch/2019/05/13/ipcc-2019-refinement/

[8] Igos E. and al. et Life Cycle Assessment of water treatment: $i$ What is the contribution of infrastructure and operation at unit process level. [Journal]. - [s.l.] : Journal of Cleaner Production, 2014. - Vol. 65. 24- 431.

[9] Lundie S. and ál. Et al. Life Cycle Assessment for Sustainable Metropolitan Water Systems Planning. Environ. Sci. Technol. 2004, 38, 13, 3465-3473, May 21, (2004). https://doi.org/10.1021/es034206m

[10] Lundin M.; Morrison G. A life cycle assessment based procedure for development of environmental sustainability indicators for urban water systems. ELSEVIER, 2002. - Vol. 4. 145-152

[11] Mohamed-Z Messaoud-Boureghda and ál. et The study of potable water treatment process in Algeria (boudouaou station) by the application of life cycle assessment (LCA). [Journal]. [s.1.] : Journal of Environmental Health Science \& Engineering, 2013. - 37 : Vol. 11. - ISSN: 2052-336.

[12] Pinto Hernández JE, Salazar de Cardona M. Cambio climatico y vulnerabilidad: prospectivas para la region nororiental de colombia - santanderes. Bistua:Revista de la Facultad de Ciencias Basicas. 12 (1) (2014) 16-45. http://revistas.unipamplona.edu.co/ojs viceinves/index.php/BIS TUA/article/view/1817/807

[13] Schulze C. Et ál. Comparison of Different Life-Cycle lmpact Assessment Methods for Aquatic Ecotoxicity. Environmental Toxicology and Chemistry, 2000. - 9 : Vol. 20. 2122-2132

[14] Slagstad H. and Brattebo H. Life cycle assessment of the water and wastewater system in Trondheim, Norway - A case study. Urban Water Journal., 2013. - Vol. 11. 23-334.

[15] Vince F. and ál. et LCA tool for the environmental evaluation of potable water production. Elsevier. Desalination, 2007. - Vol. 220. 37-56. 